\title{
Combining High Porosity with High Surface Area in Flexible Interconnected Nanowire Meshes for Hydrogen Generation and Beyond
}

Stanislaw P. Zankowski ${ }^{a, b}{ }^{*}$ and Philippe M. Vereecken ${ }^{a, b}$

a imec, Kapeldreef 75, Leuven 3001, Belgium

${ }^{\mathrm{b}}$ University of Leuven (KUL), Centre for Surface Chemistry and Catalysis, Kasteelpark Arenberg 23, Leuven 3001, Belgium

*stanislaw.zankowski@imec.be

Keywords: interconnected nanowires, current collector, flexibility, surface area, porosity, hydrogen evolution reaction 


\begin{abstract}
Nanostructured metals with large surface area have a great potential for multiple device applications. Although various metal architectures based on metal nanoligaments and nanowires are well known, they typically show a tradeoff between mechanical robustness, high surface area and high (macro)porosity, which, when combined, could significantly improve the performance of devices such as batteries, electrolyzers or sensors. In this work we rationally designed templated networks of interconnected metal nanowires, combining for the first time high porosity of metal foams, narrowly-distributed macropores and a very high surface area of nanoporous dealloyed metals. Thanks to their structural uniformity, the few-micron thick nanowire meshes are also remarkably flexible and durable. We show how the textural properties of the material can be precisely tuned to optimize the nanowire networks for applications in different devices. In an exemplary application in electrolytic production of hydrogen, thanks to its high surface area, a few-micron thick nanomesh outperformed a 300 times thicker nickel foam. Furthermore, thanks to its high porosity, the Pt-doped nanomesh surpassed a microporous $\mathrm{Pt} / \mathrm{C}$ cloth, demonstrating benefits of the optimally designed nanowire structure for a simultaneous improvement and miniaturization of electrochemical devices. This work extends the potential of interconnected nanowires to multiple new research and industrial applications requiring highly porous and flexible conductive materials with high surface-to-volume ratio.
\end{abstract}




\section{Introduction}

Porous metals with high surface area are broadly used as structural current collectors in multiple applications, such as catalysis, ${ }^{1}$ filtration, ${ }^{2}$ fuel cells,${ }^{3}$ batteries, ${ }^{4}$ supercapacitors,${ }^{5}$ electrolyzers ${ }^{6}$ or sensors. ${ }^{7}$ On the one hand, the high porosity of the porous metal is desired to accommodate greater volume of functional materials (for example, energy-storing components in high capacity batteries) or molecules (e.g. gas in fuel cell electrodes). On the other hand, the high surface area of the metal can enable higher reaction rates and lower internal resistance within the device, improving, for example, charging time of a battery ${ }^{8}$ or sensitivity of a sensor. ${ }^{9}$ Importantly, high volumetric surface area of the metal (in this paper abbreviated as VSA, also known as surface-tovolume ratio) allows to shrink the size of a porous current collector while keeping its total surface area high, facilitating device miniaturization and integration in e.g. portable electronics, intravascular sensors or non-invasive implants. ${ }^{10-13}$

Table 1. Typical properties of nanoporous gold and metal aerogels ${ }^{a}$

\begin{tabular}{lll}
\hline & Nanoporous gold & Metal aerogels \\
\hline$V S A\left(\mathrm{~m}^{2} / \mathrm{cm}^{3}\right)^{b}$ & $40-80$ & $0.5-6.5$ \\
Porosity $(\%)$ & $60-80$ & $97-99+$ \\
$d_{\text {pore }}(\mathrm{nm})^{c}$ & $5-40$ & $10-100$ \\
Robustness & brittle & brittle \\
\hline
\end{tabular}

${ }^{a}$ Data based on Refs. ${ }^{14,15} \cdot{ }^{b} V S A$ conversion is presented in Supporting Information. ${ }^{c} d_{\text {pore }}-$ effective pore diameter.

Various recent studies have shown that rather than having very high surface area but low porosity and small pores, an optimal, mechanically stable current collector should exhibit a balanced combination of high surface area and high porosity, together with sufficiently large, interconnected and preferably ordered pores, whose optimal size depends on the particular application. ${ }^{16-19}$ Currently, however, even the state-of-the-art porous metals, such as dealloyed metals 
(e.g. nanoporous gold $)^{14}$ or metal aerogels ${ }^{15}$ show a tradeoff between their individual textural properties (porosity, pore size, VSA) and mechanical stability (Table 1; for a detailed comparison, see Results and Discussion below). To a big extent, such a tradeoff is a consequence of the random microstructure of these materials. ${ }^{20}$ To overcome that, structural ordering can be introduced by fabricating three-dimensional metal nanostructures inside the templates of, for example, 3Dporous anodic aluminum oxide (AAO), which can be made by anodization of aluminum alloys..$^{21,22}$ Networks of interconnected metal nanowires prepared from 3D-AAO have been known since 2012, when Wang et al. demonstrated their synthesis and application as electrodes for lithium-ion microbatteries. ${ }^{23}$ Since then, the same group reported applications of templated $\mathrm{Ni}, \mathrm{Cu}$ and carbon nanonetworks in energy storage and water condensation, ${ }^{24-26}$ while Martin et al. showed the photonic crystal behavior of their $\mathrm{Bi}_{2} \mathrm{Te}_{3}$ and polymer counterparts. ${ }^{27}$ While having extended surface area, the structure of previously reported large-diameter interconnected nanowires (thus with moderate-to-low porosity) could be further improved for the current collecting applications which require a combination of high surface area and high porosity ${ }^{16-19}$ (see e.g. the results of Wang et al., where the thickness and capacity of energy storing $\mathrm{TiO}_{2}$ coated on nickel nanowires is limited by the large diameter of the metal nanowires). ${ }^{23}$ Also, characterization of adjustable textural properties of interconnected nanowires is needed to show their extended potential for a broad range of electrochemical applications.

In this work, by rationally designing the architecture of the nanowire network, we fabricated interconnected, macroporous nanowire meshes (here, for simplicity, referred as nanomeshes) with a tunable combination of high porosity of metal foams and very high volumetric surface area of nanoporous dealloyed metals. Such combination is optimal for many device applications, as well as unique with respect to the properties of over 70 porous metals reported in literature. By 
modifying the template technique, we made remarkably flexible and durable nanomesh foils, retaining their interconnected nature upon extensive folding. Through the application of a fewmicron thick nickel nanomesh in electrolytic hydrogen production we demonstrate that the combination of the properties in the material allows it to reduce the size of a current collector by over 300 times, with more than 10-fold improvement of its electrochemical performance.

\section{RESULTS AND DISCUSSION}

2.1. Rational design and fabrication of nanomesh networks. At the beginning of the work, we fabricated nickel nanomeshes supported on rigid substrates. To produce 3D-porous AAO templates, we anodized copper-doped aluminum layers on TiN-coated silicon wafers (for the fabrication scheme, see Figure S1 in Supporting Information). To enable both high surface area and high porosity in the nanowire meshes, the pores in the 3D AAO templates need to be optimally separated and sufficiently narrow, to ensure low diameter of the nanowires with respect to their separation. The first requirement was achieved by performing the anodization in oxalic acid at $40 \mathrm{~V}$, which results in the moderate interpore distance of about $100 \mathrm{~nm} .^{28}$ When done in oxalic acid, the anodization at $40 \mathrm{~V}$ additionally increases ordering of the AAO pores, ${ }^{29}$ consequently improving regularity of the templated structure. To preserve low diameter of the AAO pores, we refrained from a commonly used pore widening step after anodization. Such pore widening, also used in the work of the other groups, is typically applied in the AAO templating to smoothen and,

in some cases, open the AAO pores. ${ }^{25,30}$ However, it also results in widening of the template pores, consequently increasing the diameter of the produced nanowires. Our change, therefore, allows to maintain small thickness of the nanowires with respect to their separation, which, as we further demonstrate, is the key in enabling high porosity of the templated material. 

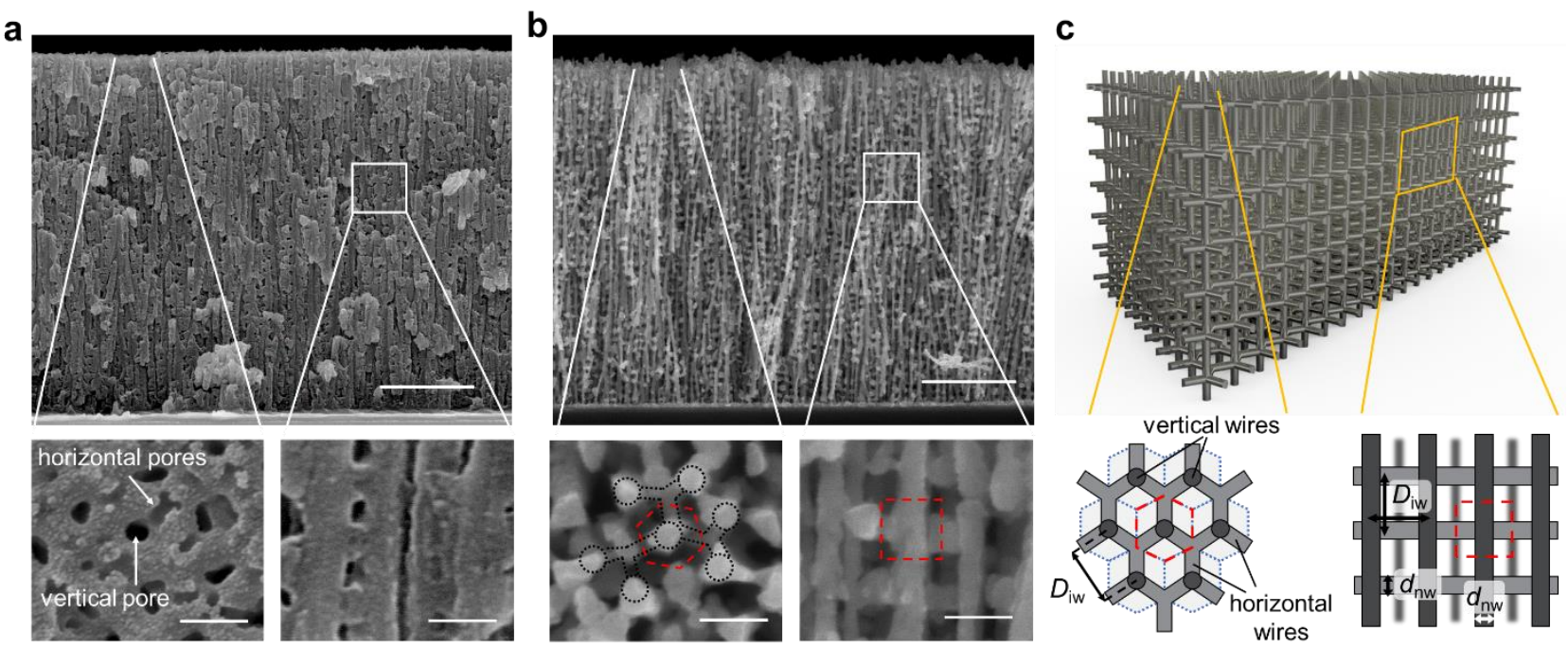

Figure 1. Nickel nanomeshes fabricated from 3D-porous templates. (a) SEM cross sectional image of the 3D-porous AAO template, together with the top view and cross section of the template in high magnification. (b) SEM cross sectional image of the nickel nanomesh after template removal, together with the top view and cross section of the mesh in high magnification. (c) Geometrical model of the nanomesh network showing the top view and cross-section. The dashed red lines in (b) and (c) represent the nanomesh unit cell and the dotted blue lines in (c) top view represent the hexagonal arrangement of the AAO vertical pores. The annotated dimensions are: $D_{\mathrm{iw}}-$ center-to-center interwire distance, $d_{\mathrm{nw}}-$ nanowire diameter. Scale bars in (a,b): $1 \mu \mathrm{m}$ (low magnification), $100 \mathrm{~nm}$ (high magnification).

The anodization performed in this manner produced AAO templates with repetitive vertical and horizontal pores, visible in the Scanning Electron Microscopy (SEM) images in Figure 1a. The horizontal openings originate from the periodic accumulation and depletion of copper atoms at the triple points between adjacent vertical pores during formation of the template. ${ }^{22}$ After anodization, nickel was electrodeposited inside the template, forming 3D nanomesh attached to the TiN bottom electrode. Following removal of the template, the structure of the nanomesh was characterized with SEM and is presented in Figure 1b. The material consists of a three-dimensional framework of interconnected vertical and horizontal nanowires grouped in periodic domains (see the insets in Figure 1b), whose geometrical architecture is the consequence of the ordered pore structure of the 3D AAO template (Figure 1a). ${ }^{22}$ Thanks to the narrow AAO pores, the nanowires exhibit small average diameter $d_{\mathrm{nw}}$ of $40 \mathrm{~nm}$ with respect to their center-to-center distance $D_{\mathrm{iw}}$ of $104 \mathrm{~nm}$. Consequently, the nanomesh framework shows a mean pore size of $64 \mathrm{~nm}$, defined by the spacing between the surfaces of neighboring wires (for the histograms, see Figure S2). The mesopores 
$(15-50 \mathrm{~nm})$ constitute $22 \%$ of the total nanomesh porosity while the remaining $78 \%$ of the porosity can be ascribed to small macropores $(50-120 \mathrm{~nm})$. The shape and size uniformity of the nanomesh pores is relatively better than of the nanoporous dealloyed metals $\mathrm{s}^{31,32}$ and significantly better than of the previously reported metal aerogels, whose pore size in some cases spans over 6 orders of magnitude, from a few nanometers up to millimeters. ${ }^{15}$ The structural uniformity of the nanowire network, also visible in the low magnification SEM images (Figure S3), is the result of a regular, electrical potential-controlled arrangement of the AAO pores,${ }^{28}$ compared to the random, capillary- and diffusion-driven pore ordering of the nanoporous metals and aerogels formed during dealloying, ${ }^{32}$ pyrolysis ${ }^{15,33}$ or liquid-phase synthesis. ${ }^{34,35}$ We note that the pore uniformity of the nanomesh could be further increased by using, for example, doubly-anodized AAO templates.

Following the recently reported spatial distribution of the 3D-AAO pores, ${ }^{22}$ the organized nanomesh microstructure can be represented by a periodic network of interconnected cylindrical wires with a diameter $d_{\mathrm{nw}}$, grouped in hexagonal unit cells (Figure 1c and Figure S4). The vertical and lateral size of each unit cell is defined by the average interwire distance $D_{\text {iw }}$. The vertical wires are interconnected via the honeycomb lattice of the horizontal wires, originating from the hexagonal distribution of copper impurities during the formation of 3D AAO templates. ${ }^{21,22}$ This is also partially evidenced in the top view insets of Figure 1a-b, showing the horizontal AAO pores meeting at $\sim 120^{\circ}$, and the corresponding nanowire structure. The hexagonal arrangement of the horizontal wires lowers the density of the nanomesh ${ }^{36}$ simultaneously stabilizing the nanowires framework and increasing its resistance to fracture.

2.2. Mechanical flexibility. To demonstrate mechanical properties of the interconnected nanowire material, we fabricated free-standing foils of $\sim 4.5 \mu \mathrm{m}$ thick nanomesh supported by a 


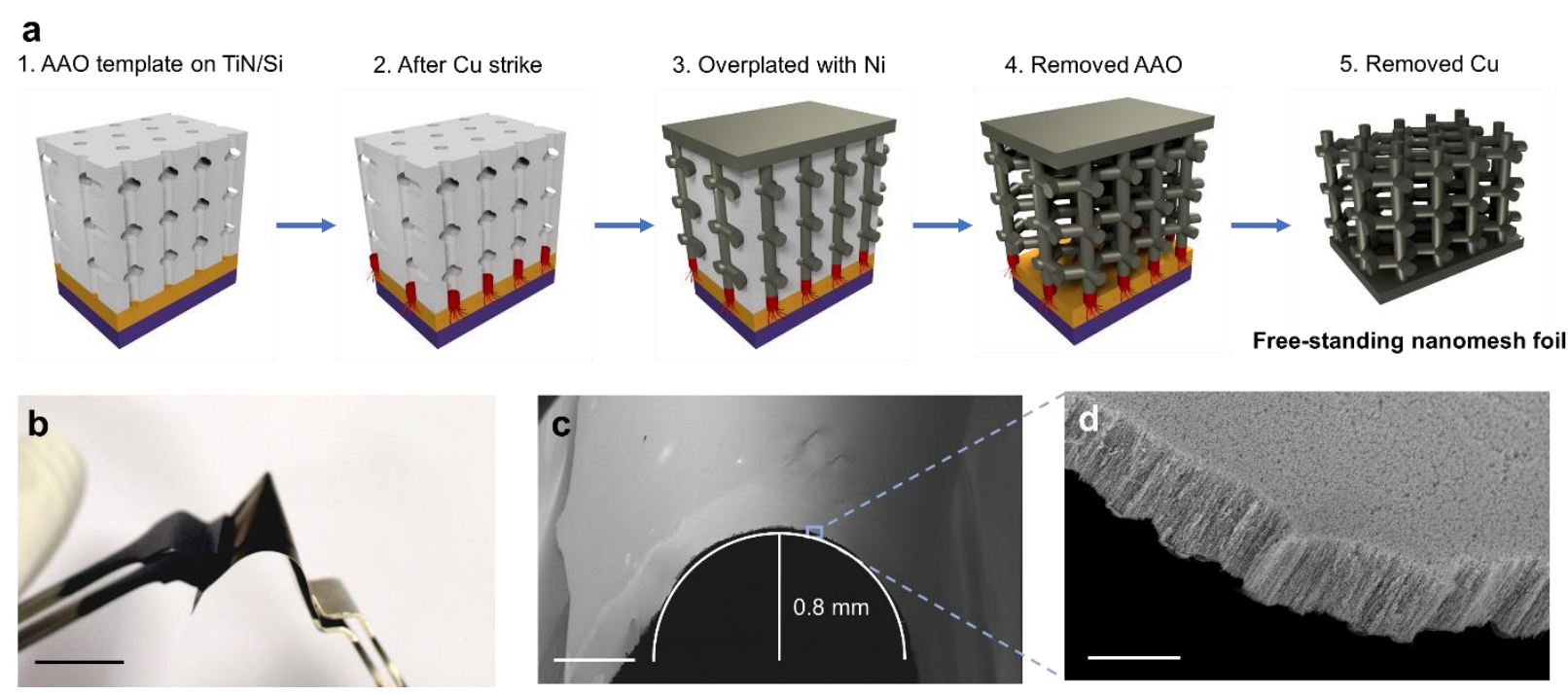

Figure 2. Flexibility of the nanomesh. (a) Fabrication scheme of flexible nanomesh foils from AAO/TiN/Si wafers. (b) Photograph of the flexible nanomesh sheet. (c, d) Scanning electron micrographs of the edge of the nanomesh bent with a radius of $\sim 0.8 \mathrm{~mm}$ in low (c) and high (d) magnification. The self-supported nanomesh retains its interconnected nature and ordering during large deformations. Scale bars: (b) $-2 \mathrm{~cm},(\mathrm{c})-500 \mu \mathrm{m},(\mathrm{d})-5 \mu \mathrm{m}$.

few-micron thick nickel film (Figure 2). Such foils were obtained by overplating the metal on top of the AAO templates and separating the material from the wafer substrate. The separation was achieved by introducing a sacrificial copper strike at the bottom of the AAO templates prior to nickel electrodeposition, as schematically presented in Figure 2a. This additional step, upon selective etching of copper, ensured a uniform detachment of the network from the rigid wafer. The planar nickel layer, now at the bottom of the nanowires, helps to stabilize the framework, providing at the same time good electrical contact to the entire material. Remarkably, the freestanding nanomesh foils are both durable and flexible; repetitive folding about a submillimeter radius did not induce any observable damage to the networks in either macro- or microscale (Figure 2b-d). This is in sharp contrast to the typical brittleness of nanoporous dealloyed metals and metal aerogels, where a single crack in their random microstructure can lead to a catastrophic breakdown of the material. ${ }^{37}$ Besides the small thickness of the nanomesh, its excellent durability upon flexing can be associated with the ordered character of the framework. 
Because the pores in the 3D-AAO template are uniformly distributed, all the nanowires are equally interconnected and form a monolithic, crack-free network. Also, during folding, the distances between the nanowires are four orders of magnitude smaller with respect to the bending radius of the foil. Thus, the bending-induced stress becomes uniformly distributed over the numerous small nanowire domains, which individually experience only a small strain.

Importantly, fabrication of flexible nanomesh which requires exposure of the nanowires to both $\mathrm{KOH}$ and a copper etchant does not change the metallic nature of the bulk of the nanowires, as verified with grazing incidence X-ray diffraction (Figure S5). The diffraction pattern shows exclusively peaks corresponding to nickel (111), (200) and (220) crystallographic planes. Nonetheless, the surface of the nanowires is likely passivated with a thin $(<2 \mathrm{~nm})$ amorphous nickel oxide/hydroxide layer, as a result of exposure of the material to the etchants and air. ${ }^{38,39}$

2.3. Combining high porosity with high surface area. To assess textural properties of the nanomesh, we applied a combination of SEM, electrochemical and gas adsorption methods on the nanomeshes with increasing total thickness of the networks (see Experimental Section and Figures S6 and S7). Thus, the porosity of the nanomesh was determined as $76 \pm 0.5 \%$, while the VSA was found as $26 \pm 2 \mathrm{~m}^{2} / \mathrm{cm}^{3}$ (electrochemical) and $20 \mathrm{~m}^{2} / \mathrm{cm}^{3}$ (BET $\mathrm{Kr}$ adsorption). To understand the origin of such unusual combination of high porosity and high surface area in the macroporous material, we derived mathematical expressions for porosity and VSA for the ideal nanomesh structure with a given nanowire diameter and spacing (the derivation is given in Supplementary Materials). The theoretical porosity $P$ was found to depend, in fact, only on the nanowire spacing-to-diameter ratio $\gamma=D_{i w} / d_{n w}$ while the model $V S A$ is additionally proportional to the inverse nanowire spacing $D_{i w}$, as described by Equations 1 and 2, respectively: 
$P=100 \% \times\left(1-\frac{\gamma \times \pi(3+\sqrt{3})-9}{6 \gamma^{3}}\right)$

$V S A=\frac{\gamma \times 2 \pi(3+\sqrt{3})-27}{3 \gamma^{2} \times D_{\mathrm{iw}}}$

By using the mean nanowire diameter and spacing, both previously determined with SEM, we calculated the model porosity as $72 \pm 4 \%$ and the model $V S A$ as $24 \pm 2 \mathrm{~m}^{2} / \mathrm{cm}^{3}$ - in perfect agreement with the experimental data. The matching of experimental and model properties shows that the non-trivial combination of high volumetric surface area and high porosity is a result of the uniform and ordered structure of the material, which thanks to the narrow and optimally separated AAO pores, exhibits high ratio of the interwire distance to the nanowire diameter. The rules defined in Equations 1 and 2 allow to design interconnected nanowires with a desired pore size, high surface area and, at the same time, high porosity, which is the target combination in current collecting applications.

2.4. Engineering nanomesh properties. Specific applications of a porous metal gain from further optimization of its high surface area over macroporosity (e.g. in electrocatalysis) or from increasing its macroporosity with acceptable sacrifice in surface area (e.g. in batteries) ${ }^{19}$ To gain control of the nanomesh textural properties, we modified the pore distribution of the AAO templates using two common techniques: by changing the anodization voltage or by AAO pore widening, performed in dilute $\mathrm{H}_{3} \mathrm{PO}_{4}$ after anodization. Upon deposition of nickel, both treatments led to modification of the nanomesh structure and properties, as presented in Figures 3 and summarized in Table S1 (for SEM images of all the samples, see Figure S8). On the one hand, the decrease of anodization potential from $40 \mathrm{~V}$ to $30 \mathrm{~V}$ lowered the separation between the nanowires, 
a
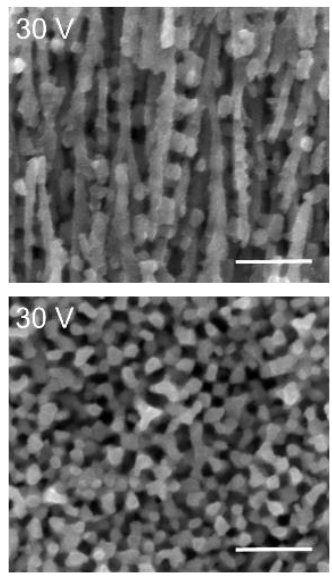

C
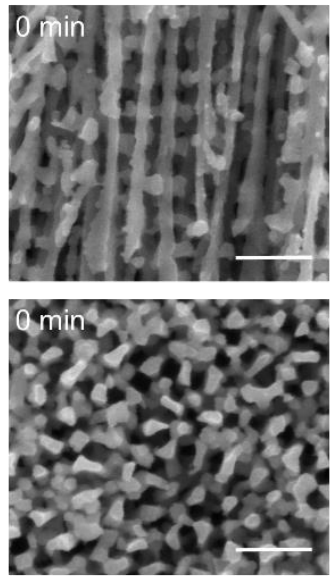
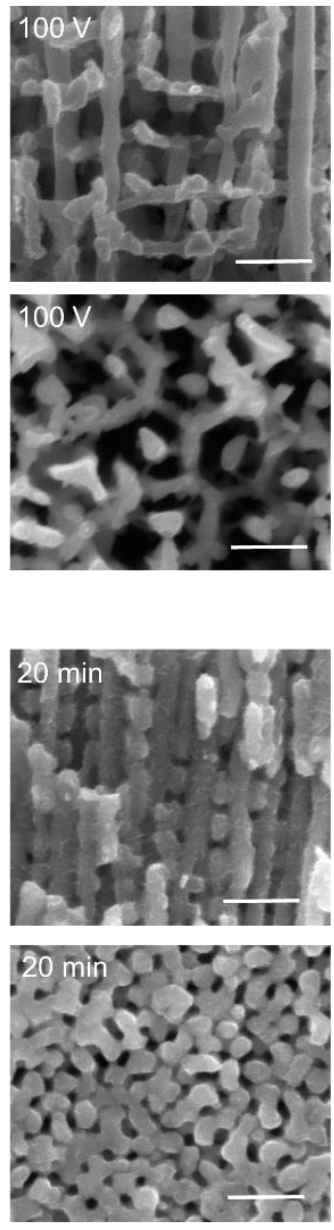

b

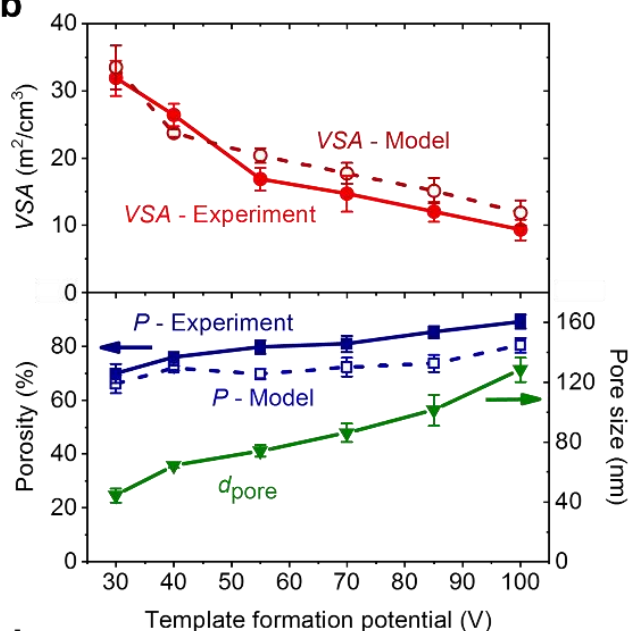

d

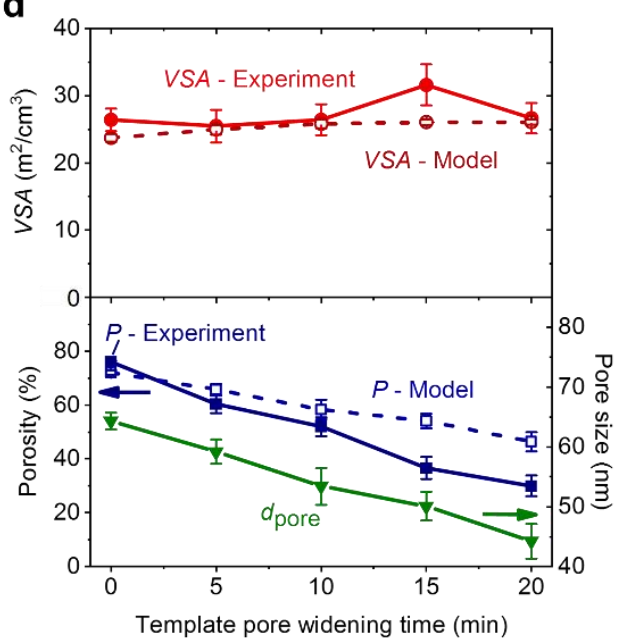

Figure 3. Engineering of the nanomesh structure. (a) SEM images of nanomeshes (upper row - cross section, lower row - top view) made from AAO templates formed at $30 \mathrm{~V}$ and $100 \mathrm{~V}$. (b) Nanomesh volumetric surface area $(V S A)$, porosity $(P)$ and pore size $\left(d_{\text {pore }}\right)$ vs. template anodization voltage. (c) SEM images of the nanomeshes (upper row - cross section, lower row top view) made from pristine $(0 \mathrm{~min})$ and pore-widened $(20 \mathrm{~min})$ AAO templates, anodized at $40 \mathrm{~V}$. (d) Nanomesh VSA, porosity and pore size vs. template pore widening time. The error bars in $(b, d)$ represent $95 \%$ confidence level of the mean values. The model values of VSA and porosity were calculated using Equations 1 and 2, with the mean nanowire diameter and spacing determined with SEM for each nanomesh. Scale bars in $(\mathrm{a}, \mathrm{c}): 200 \mathrm{~nm}$.

resulting in mesoporous nanomeshes (mean pore size of $44 \mathrm{~nm}$ ) with enlarged surface area $\left(32 \mathrm{~m}^{2} / \mathrm{cm}^{3}\right)$ and only slightly decreased porosity (70\%) (Figure $\left.3 \mathrm{a}-\mathrm{b}\right)$. On the other hand, the increase of anodization potential gave more scattered nanomeshes with larger pores (up to $128 \mathrm{~nm}$ ), higher porosity (up to $89 \%$ ) but lowered $V S A$ of $9 \mathrm{~m}^{2} / \mathrm{cm}^{3}$ for $100 \mathrm{~V}$ anodization. The pore widening, applied to the $40 \mathrm{~V}$ - anodized AAO, increased the nanowire diameter without affecting the nanowire separation, thus reducing the average pore size of the nanomesh from $64 \mathrm{~nm}$ 
to $44 \mathrm{~nm}$ after 20 min of etching (Figure 3c-d). The $V S A$ of the material remained, however, largely unaffected by the thickening of the nanowires. The geometrical model of the nanomesh also predicts a broad maximum of the VSA as the nanowire diameter approaches the nanowire separation (see also Figure S9). Such an effect can be explained by the competing increase of the $V S A$ due to the thickening of the wires and reduction of the $V S A$ due to the increasingly bulky character of the material. The increase of nanowire diameter comes with a reduction of nanomesh porosity to $50 \%$ after $10 \mathrm{~min}$ of etching. This shows that unless the ratio between nanowire separation and diameter is very large, thickening of the metal skeleton does not guarantee significant gains in surface area, but can result in a significant loss of porosity. After longer etching times, the decrease in porosity is amplified by the coalescence of some of the nanowires, visible in Figure 3c. The structure of the low porosity nanomeshes is similar to the morphology of the previously reported interconnected nanowire networks, which had reduced nanowire spacing-todiameter ratio as a result of the pre-etching of the AAO templates..$^{23,27}$

2.5. Potential applications. The changes of the nanomesh surface area and porosity are exclusively due to the modification of the mean nanowire spacing and diameter, as shown by the matching trends of the VSA and porosity predicted by the geometrical model for each of the synthesized meshes (dashed lines in Figure $3 \mathrm{~b}$ and $3 \mathrm{~d}$ ). This encouraged us to explore the broader potential of the nanomesh structure, by simulating porosity and surface area of the material with various nanowire configurations (Figure 4). We found that, on the one hand, lowering the interwire distance below $50 \mathrm{~nm}$ could allow for mesoporous (e.g. $25 \mathrm{~nm}$ ) nanomeshes with very high surface area of above $50 \mathrm{~m}^{2} / \mathrm{cm}^{3}$ and porosity above $60 \%$. Although such properties are not uncommon for nanoporous gold, the non-noble character of the interconnected nanowire networks is a 

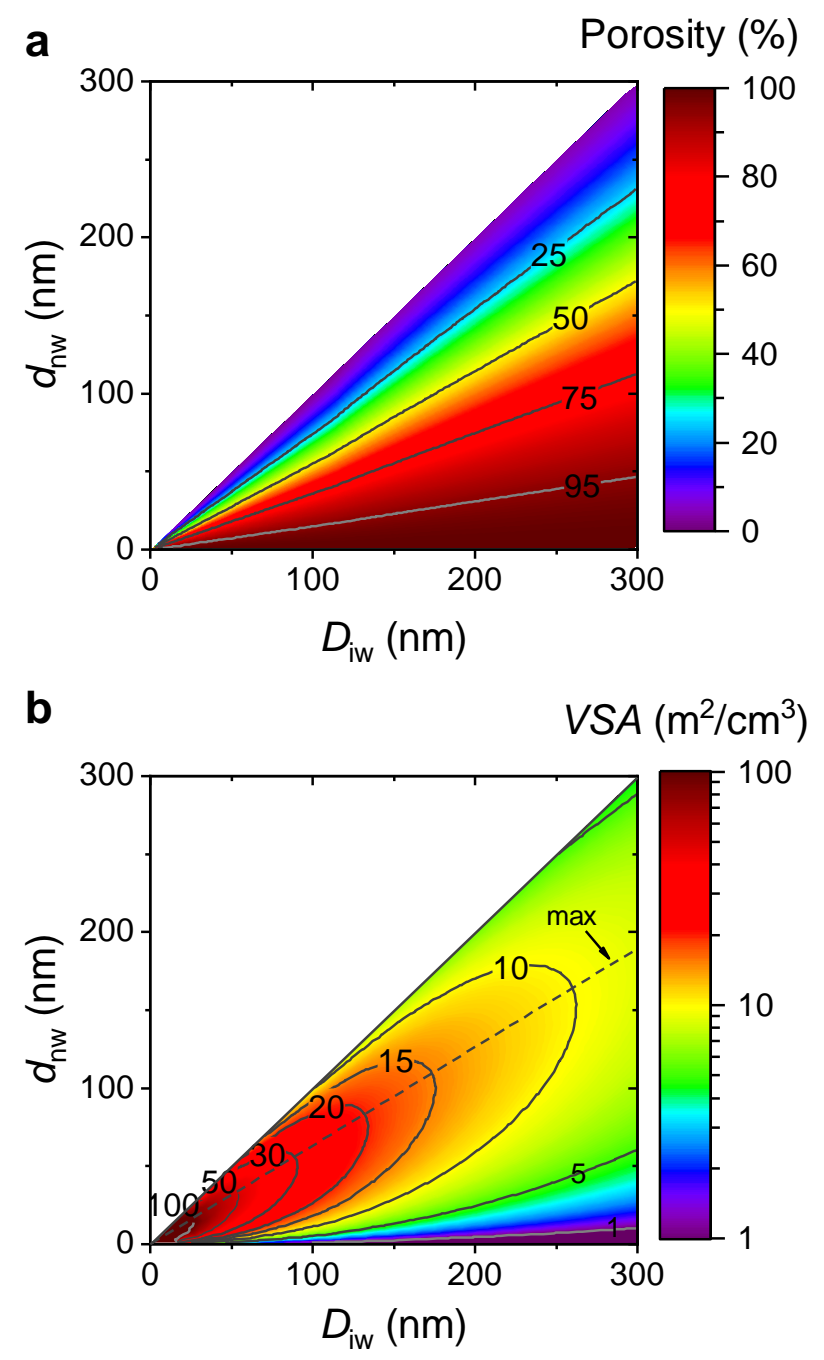

Figure 4. Modeling of nanomesh properties. (a) Nanomesh porosity as a function of nanowire diameter $d_{\mathrm{nw}}$ and spacing $D_{\text {iw. }}$ (b) Nanomesh volumetric surface area (VSA) as a function of nanowire diameter $d_{\mathrm{nw}}$ and spacing $D_{\mathrm{iw}}$. The dashed line in (b) shows the maximum VSA, characteristic for the nanomeshes with $d_{\mathrm{nw}} \approx 0.6 D_{\mathrm{iw}}$. The modeling was done based on Equations 1 and 2 .

considerable economic advantage for their use in e.g. fuel cells or electrocatalysis, where they can simultaneously act as a current collector and a functional catalyst. On the other hand, highly porous networks having pore size of more than $200 \mathrm{~nm}$, porosity above $90 \%$ and still significantly high surface area above $5 \mathrm{~m}^{2} / \mathrm{cm}^{3}$ could be produced by appropriately increasing the interwire spacing without excessive increase of nanowire diameter. ${ }^{40}$ Such nanomeshes are attractive candidates for applications in, for example, high capacity and fast charging batteries and supercapacitors, where 

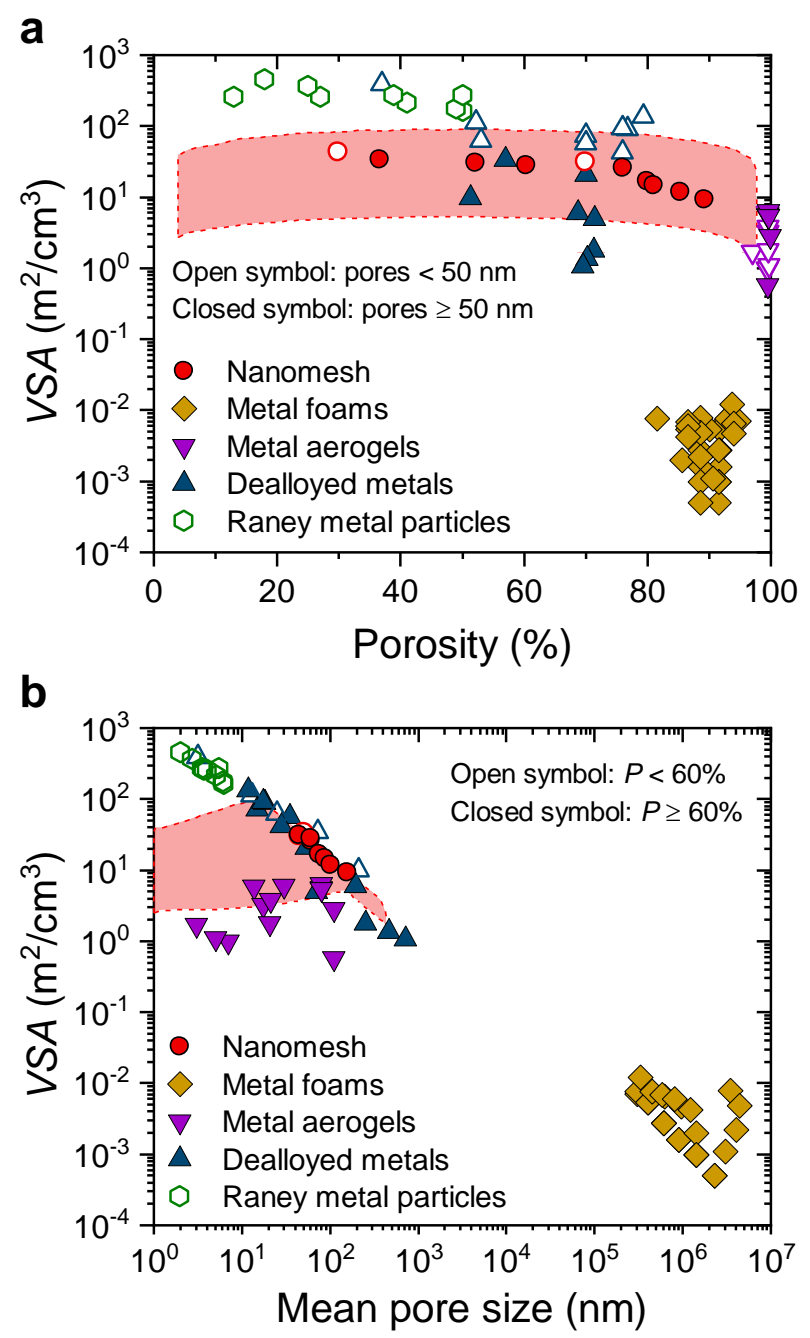

Figure 5. Comparison of the properties of the nanomesh and other porous metals. (a) Volumetric surface area (VSA) vs. porosity. (b) VSA vs. mean pore size. The open and closed symbols in (a) represent materials with mean pore size smaller and larger than $50 \mathrm{~nm}$, respectively. The open and closed symbols in (b) represent materials with porosity smaller and larger than $60 \%$, respectively. The colored-in areas represent modeled properties of the nanomesh with $D_{\text {iw }}$ from $30 \mathrm{~nm}$ to $500 \mathrm{~nm}$ and $d_{\text {nw }} \geq 0.1 D_{\text {iw }}$. The list of references is provided in Table S2 in Supporting Information.

high loading of energy-storing materials, large pores of the current collector and large contact area between both components are needed. ${ }^{18}$ The previously described maximum of the nanomesh VSA can also be observed in the simulated trends in Figure $4 \mathrm{~b}$ for the systems with $d_{\mathrm{nw}} \approx 0.6 D_{\mathrm{iw}}$. This 
simple relation allows to maximize the nanomesh surface area for each desired pore size of the material, which is important in, for example, filtering applications.

We further compared the porosity, average pore size and VSA of the nanomesh to the properties of over 70 porous metals reported in the literature (Figure 5 and Table S2). The nanomeshes can exhibit up to 100 times higher volumetric surface area compared to the metal aerogels while maintaining high porosity of metal foams (above 75\%). Even for the macroporous nanomeshes having porosity close to $90 \%$, their surface area remains higher than of the state-of-the-art macroporous dealloyed metals and metal aerogels, which is a consequence of the ordered and uniform structure of the interconnected nanowire material. Although some of the dealloyed metals exhibit higher total VSA, in such cases they show lower porosity and/or smaller pores $(13-35 \mathrm{~nm})$, which impacts their broader applicability. Also, Raney metals (most commonly nickel), a wellestablished class of catalysts in organic synthesis, have much higher surface area (up to $\left.450 \mathrm{~m}^{2} / \mathrm{cm}^{3}\right)$, but their low porosity $(<50 \%)$, very small pore size $(<10 \mathrm{~nm})$ and loose particle nature exclude their structural, current collecting applications. On the other hand, the monolithic interconnected nanowire networks offer the largest combined surface area and porosity of any of the macroporous metals in the comparison, surpassing the highest porosity of the nanoporous dealloyed metals (79\%) and the highest surface area of the metal aerogels $\left(7 \mathrm{~m}^{2} / \mathrm{cm}^{3}\right)$. Since the nanomesh thickness is bound by the achievable thickness of AAO templates - currently about 150 microns $^{41}$ - we estimate that an effective surface enhancement of more than 5000x per $\mathrm{cm}^{2}$ footprint area can be achieved, without compromising high porosity of the material.

2.6. Application in electrolytic hydrogen generation. To effectively demonstrate the benefits of the combination of high surface area and high (macro)porosity in the nanowire meshes, we used 

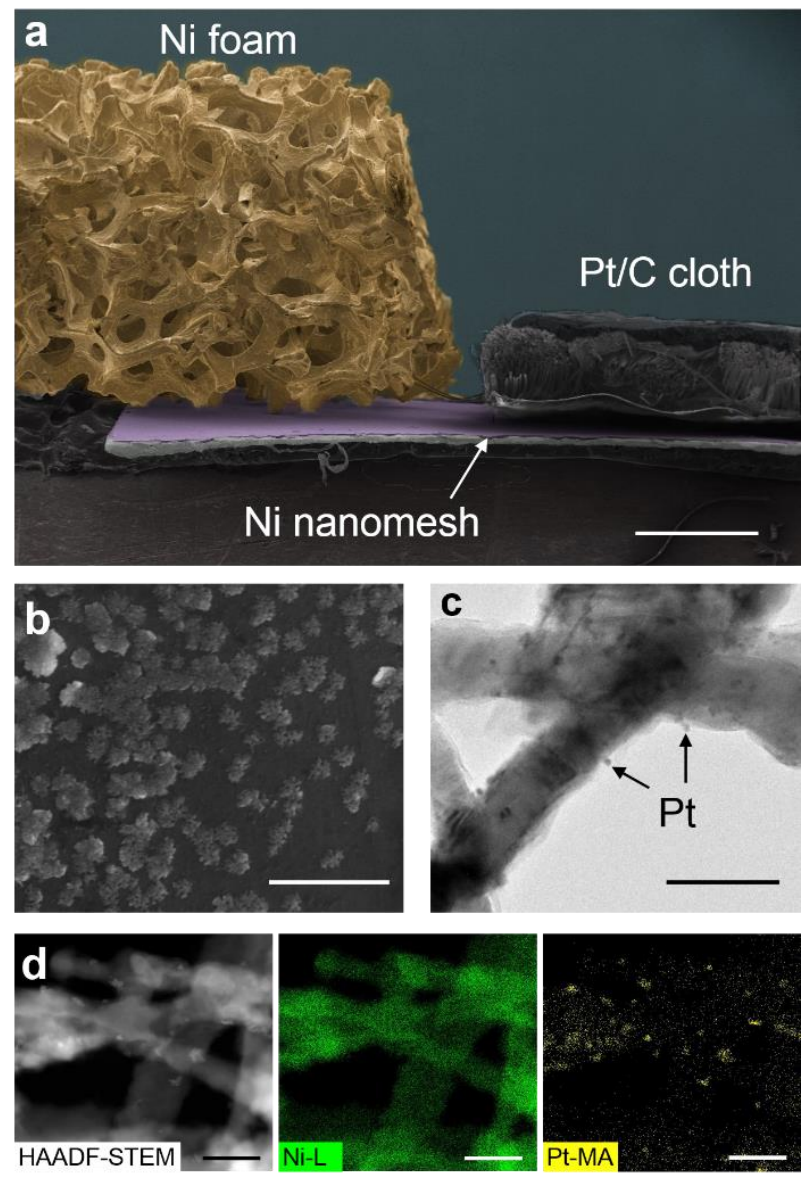

Figure 6. (a) Colour-enhanced SEM image of a $1.5 \mathrm{~mm}$ thick Ni cellular foam and a $0.41 \mathrm{~mm}$ thick $\mathrm{Pt} / \mathrm{C}$ cloth, laying on a $4.8 \mu \mathrm{m}$ thick Ni nanomesh. (b) SEM image of Ni foam surface coated with Pt nanoclusters. (c) TEM bright field image of nanowire surface after Pt coating. (d) STEM-EDS colour mapping of the nanowires for nickel (green) and platinum (yellow). The scale bars are: (a) - 500 $\mu \mathrm{m}$, (b) - $500 \mathrm{~nm}$, (c) $-50 \mathrm{~nm}$, (d) - $60 \mathrm{~nm}$.

the example of hydrogen generation, which is one of the key methods for large scale, long term energy conversion and storage. ${ }^{42}$ Accordingly, we applied the flexible $4.75 \mu \mathrm{m}$ thick nanomesh (76\% porosity, $64 \mathrm{~nm}$ pore size, $126 \mathrm{~cm}^{2}$ footprint-normalized surface area) as a cathode during hydrogen evolution reaction (HER) in $1 \mathrm{M} \mathrm{KOH}$. The performance of the thin nickel nanomesh was benchmarked against a commercial $1.5 \mathrm{~mm}$ thick high-surface nickel foam (96\% nominal porosity, $10 \mathrm{~cm}^{2}$ normalized surface area) and a PTFE-coated $\mathrm{Pt} / \mathrm{C}$ cloth $(20 \% \mathrm{wt}$. Pt $=$ $0.2 \mathrm{mg} \mathrm{Pt} / \mathrm{cm}^{2}$ ) with a $90 \mu \mathrm{m}$ thick microporous layer, both commonly used as electrodes in water 

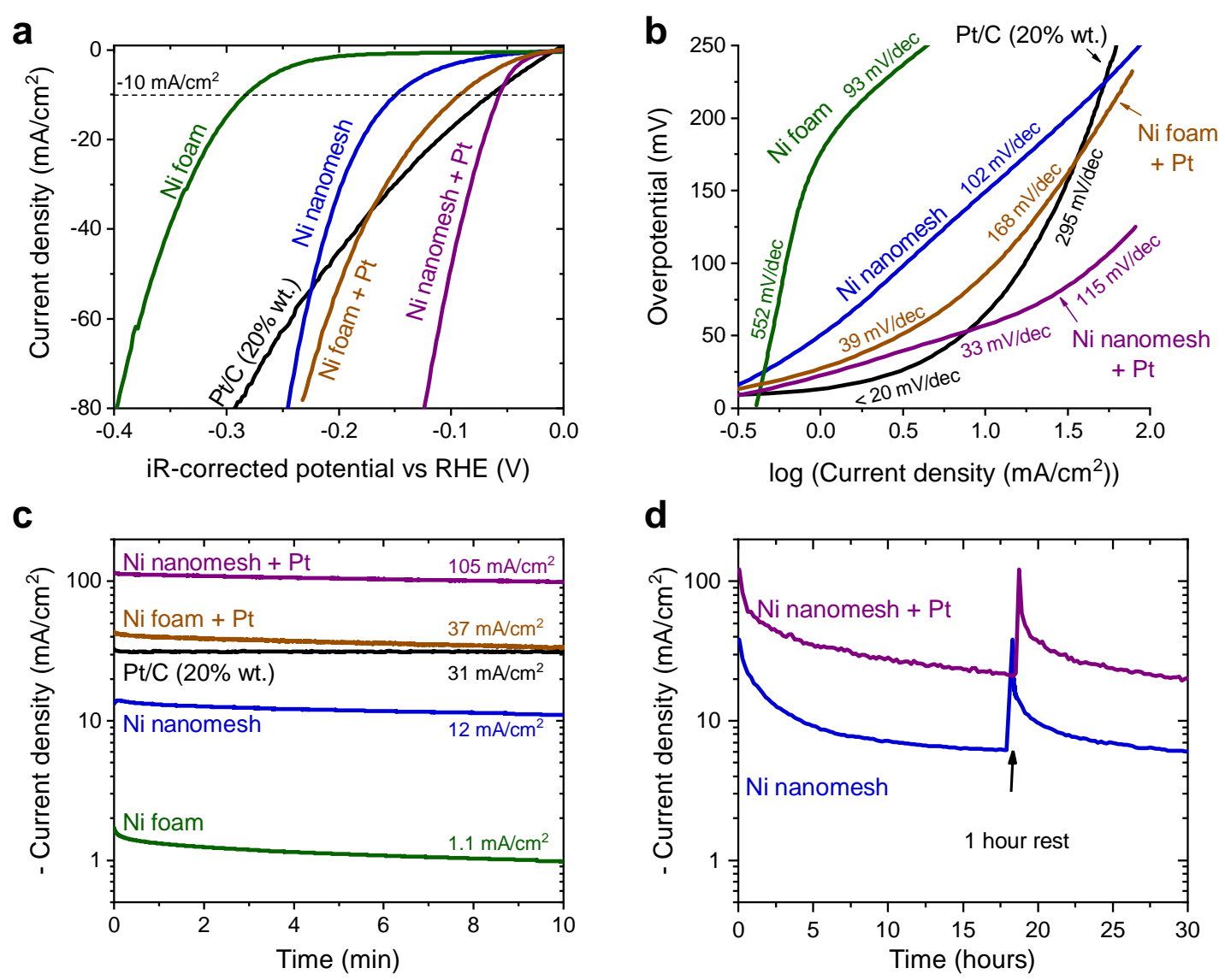

Figure 7. (a) Linear scan voltammograms of nickel electrodes and $\mathrm{Pt} / \mathrm{C}$ cloth during HER. (b) Tafel analysis of the voltammograms. (c) Current transients recorded during $10 \mathrm{~min}$ at $-0.2 \mathrm{~V}$ vs. RHE, without iR compensation. (d) Long-term stability recorded at $-0.2 \mathrm{~V}$ vs. RHE under mild electrolyte agitation, without iR compensation. All the currents are normalized to the geometrical area $\left(2 \mathrm{~cm}^{2}\right)$ of the samples.

electrolyzers (Figure 6a). To further enhance the activity of the nickel materials, after the initial HER tests they were coated with platinum catalyst, deposited by 3 cycles of chemisorption and electroreduction of hexachloroplatinic acid. Such treatment led to formation of larger Pt nanoagglomerates on the foam and small Pt nanoparticles on the mesh (Figure 6b-d). The total loading of platinum on the nanomesh was determined with Energy Dispersive X-ray spectroscopy (EDX) as $\sim 0.1 \mathrm{mg} / \mathrm{cm}^{2}$ (Figure $\mathrm{S} 10$ ) while the loading on the foam is estimated at $0.2-0.6 \%$ wt. or $0.1-0.3 \mathrm{mg} / \mathrm{cm}^{2} .{ }^{43}$ Since the total amount of Pt is in the similar range for all the tested materials, no further optimization of Pt loading was performed. 
Linear scan voltammetry was performed to assess the intrinsic performance of the materials. The measurements were done with the correction for uncompensated resistance (so-called iR drop), ${ }^{44}$ determined with impedance spectroscopy. The voltammograms, presented in Figure 7a, show that the bare nanomesh delivered significantly higher hydrogen generation currents at any overpotential, as compared to the bare nickel foam. Thanks to its very high surface area and good electrical conductivity, the thin nanomesh reached a current of $10 \mathrm{~mA} / \mathrm{cm}^{2}$ at $149 \mathrm{mV}$ of overpotential. Such overpotential is not only $133 \mathrm{mV}$ less than that for the thick nickel foam $(282 \mathrm{mV})$, but also less than for the previously reported nanoporous nickel $(170 \mathrm{mV}),{ }^{45}$ comparable to the state-of-the-art, non-noble catalysts such as Co-P/NCs $(154 \mathrm{mV})^{46}$ or $\mathrm{MoC}_{\mathrm{x}}$ $(151 \mathrm{mV}),{ }^{47}$ but somewhat higher than e.g. $\mathrm{Mo}_{1} \mathrm{~N}_{1} \mathrm{C}_{2}(132 \mathrm{mV}),{ }^{48} \mathrm{Cu}$ NDs $/ \mathrm{Ni}_{3} \mathrm{~S}_{2}$ NT-CFs $(128 \mathrm{mV}),{ }^{49} \mathrm{TiO}_{2} \mathrm{NDs} / \mathrm{Co}$ NSNTs-CFs $(108 \mathrm{mV}){ }^{50}$ or W-SAC $(85 \mathrm{mV}) .{ }^{51}$ We can note, however, that the preparation of such catalysts typically requires multistep synthesis at high temperatures or pressures, followed by their loading onto millimeter-thick conductive supports such as nickel foams or carbon fabrics. Compared to the nickel foam, the nanomesh shows much lower polarization resistance at moderate overpotentials, demonstrated by the Tafel slope of $102 \mathrm{mV} / \mathrm{dec}$ (nanomesh), as compared to $552 \mathrm{mV} / \mathrm{dec}$ (Ni foam) (Figure 7b). Upon functionalization of both materials with platinum, their activity towards hydrogen generation significantly increased, reducing the overpotential required to drive $10 \mathrm{~mA} / \mathrm{cm}^{2}$ of current down to $91 \mathrm{mV}$ (nickel foam) and only $57 \mathrm{mV}$ (nanomesh). In the low overpotential region, the microporous platinized carbon showed similar performance to the platinized nanomesh, reaching $10 \mathrm{~mA} / \mathrm{cm}^{2}$ at $65 \mathrm{mV}$.

To assess the power performance of the electrodes, we polarized them for 10 min at a constant overpotential of $200 \mathrm{mV}$ and measured the average current (Figure 7c). No correction for the iR drop was applied this time. The few-micron thick nanomesh delivered an average current of 
$12 \mathrm{~mA} / \mathrm{cm}^{2}$, marking $11 \mathrm{x}$ increase over the current recorded on the millimeter-thick nickel foam $\left(1.1 \mathrm{~mA} / \mathrm{cm}^{2}\right)$, which is a clear result of an order of magnitude higher surface area of the nanomesh. After coating with platinum, both the foam and the nanomesh show significantly higher average currents $\left(37 \mathrm{~mA} / \mathrm{cm}^{2}\right.$ and $105 \mathrm{~mA} / \mathrm{cm}^{2}$, respectively). At high overpotential, the modified nanomesh outperformed platinized carbon cloth $\left(31 \mathrm{~mA} / \mathrm{cm}^{2}\right)$ (also see the photograph in Figure S11). The superior power performance of the nanomesh can be ascribed to the lower sheet and diffusion resistance $(<300 \mathrm{~m} \Omega)$ within the regular macroporous metal network, as compared to the resistance of the random microporous structure of the carbon material $(\sim 1 \Omega)$. This demonstrates that the combination of high surface area and large (macro)porosity within the nanomesh current collector can lead to significantly increased performance of an electrode, with simultaneous reduction (in our example, up to 300 times) of its size.

Finally, long-term stability was assessed for freshly prepared pristine and platinum-coated nanomesh samples, during 30 hours of HER at $-0.2 \mathrm{~V} v s$. RHE under mild agitation (Figure 7d). Both the nanomesh samples exhibit initially high currents which decreased during the first 6 hours of experiment to $8.5 \mathrm{~mA} / \mathrm{cm}^{2}$ (pristine nanomesh) and $33 \mathrm{~mA} / \mathrm{cm}^{2}$ (platinized nanomesh) respectively. This was followed by a steady state regime with an average current of $6.5 \mathrm{~mA} / \mathrm{cm}^{2}$ (pristine nanomesh) and $26 \mathrm{~mA} / \mathrm{cm}^{2}$ (platinized nanomesh) during the next 12 hours of operation. Although these values are significantly lower than the initial currents, they are still close to or better than the currents reached on the considerably thicker nickel foam and $\mathrm{Pt} / \mathrm{C}$ cloth in the shortterm experiment. At the current stage, we believe that deactivation of the nanomesh during prolonged HER could be a result of poisoning of the nanomesh with surface-adsorbed hydrogen and formation of passive $\mathrm{NiH}_{\mathrm{x}}$ (likely accelerated by the high surface area of the nickel nanowires) or from the saturation of the nanomesh pores with $\mathrm{H}_{2}$ bubbles. Both the effects were previously 
shown to significantly increase resistance of nickel electrodes during extended HER ${ }^{52-55}$ Presence of $\mathrm{NiH}_{\mathrm{x}}$ on the nanomesh was also detected by cyclic voltammetry after long-term hydrogen generation (Figure S12). To see whether the activity of the material could be restored, the HER measurement was stopped after 18 hours, allowing the electrodes to rest at their open circuit potential for 1 hour. When the constant potential was reapplied to the electrodes, the catalytic activity of both samples was restored and the currents returned to their initially high values, followed by a similar decrease as in the first part of the experiment. This shows that although the formation of $\mathrm{NiH}_{\mathrm{x}}$ and buildup of residual gas inside the nanomesh may lower its long-term HER activity, the nanomesh performance can be renewed for the prolonged hydrogen evolution. Further optimization of the nanowire networks may improve their long-term efficiency for hydrogen generation.

\section{CONCLUSIONS}

In summary, we have shown that the rational design of interconnected nanowire meshes allows for a unique combination of high porosity, high surface area, narrow pore size distribution and mechanical flexibility within a single macroporous metal. Benefitting from the structural uniformity of the networks, the properties of the nanowire meshes depend exclusively on the mean nanowire diameter and separation. Thus, common AAO template modification techniques can be used to increase the nanomesh pore size above $100 \mathrm{~nm}$, surface area above $30 \mathrm{~m}^{2} / \mathrm{cm}^{3}$ or porosity beyond $85 \%$. When applied in electrolytic hydrogen production, because of its high surface area, a micron-thick nickel nanomesh outperformed a millimeter-thick nickel foam and, because of its high (macro)porosity, the Pt-coated nanomesh reached better power performance then the microporous platinized carbon cloth. While due to the surface poisoning the nanomesh 
performance decreased during prolonged hydrogen evolution, the activity of the material could be restored after 1 hour of resting period. Not limited to water electrolysis, the optimized interconnected nanowire current collectors can also extend their applicability in e.g. batteries, fuel cells or sensors, increasing performance of these devices while reducing their size. The versatility of template-assisted deposition also allows for the fabrication of highly porous nanomeshes or nanomesh composites made of different metals, such as silver or copper, but also semiconductors or polymers. The findings reported herein can thus bring new research interest and applications of ordered, interconnected nanowire materials.

\section{EXPERIMENTAL SECTION}

All the reported current and charge densities are normalized to the footprint area of the samples. The preparation and electrochemical characterization of all the samples was performed in the laboratory with controlled air temperature at $21.5 \pm 1{ }^{\circ} \mathrm{C}$.

Fabrication of 3D-AAO templates. The templates for fabrication of nickel nanomeshes were prepared by anodizing coupons of a wafer stack: $4 \mu \mathrm{m}$ aluminum layer doped with $0.22 \%$ at. $\mathrm{Cu}$, PVD-sputtered on a $150 \mathrm{~nm}$ PVD-sputtered-TiN/Si wafer. To fabricate wafer-supported meshes, the samples were placed on a heat exchanging aluminum block and covered with a PDMS o-ring with $18 \mathrm{~mm}$ inner diameter. On top of the o-ring, a $10 \mathrm{~mL}$ cylindrical glass cell was placed and secured using ball joint clamp. To fabricate self-supported nanomesh foils, the wafer coupons were fully immersed in a $200 \mathrm{~mL}$ jacketed cylindrical glass cell. The AAO templates were formed in $0.3 \mathrm{M}$ oxalic acid ( $\geq 99 \%$, Sigma Aldrich). Unless stated otherwise, the anodization was carried out at $30{ }^{\circ} \mathrm{C}$ and $40 \mathrm{~V}$, provided by Autolab PGSTAT100 potentiostat/galvanostat. During the 
processing, the electrolyte was stirred using mechanical stirrer operating at $1900 \mathrm{rpm}$. The anodization was carried out until the total charge passed reached the value of $11 \mathrm{C} / \mathrm{cm}^{2}$, after which rapid decline in current was normally observed, indicating complete oxidation of the aluminum layer. After this point, the anodization was continued for $500 \mathrm{~s}$ (for the wafer-supported nanomesh) or $300 \mathrm{~s}$ (for the self-supported nanomesh) to ensure breaching of the AAO barrier layer at the bottom of the pores. The $\mathrm{TiO}_{2}$ layer formed at the $\mathrm{AAO} / \mathrm{TiN}$ interface during overanodization was removed according to the procedure of Park et al. ${ }^{56}$ The sample was immersed in a solution of 1:1:5 volumetric ratio of $30 \% \mathrm{H}_{2} \mathrm{O}_{2}(\mathrm{KMG}): 29 \% \mathrm{NH}_{3(\mathrm{aq})}(\mathrm{KMG}): \mathrm{H}_{2} \mathrm{O}$ at $30^{\circ} \mathrm{C}$ for $4.5 \mathrm{~min}$ (wafer supported nanomesh) or $3.5 \mathrm{~min}$ (self-supported nanomesh), followed by rinsing with water.

Fabrication of nickel nanomeshes. The nickel nanomeshes were formed by galvanostatic deposition of nickel in the AAO templates from $0.62 \mathrm{M}$ nickel(II) sulfamate $(65 \%, \mathrm{KMG})+$ $0.62 \mathrm{M}$ boric acid (99.8\%-101\%, VWR chemicals) bath at $-10 \mathrm{~mA} / \mathrm{cm}^{2}$ and $30{ }^{\circ} \mathrm{C}$, using nickel foil (99.9\% purity, Sigma-Aldrich) as a counter electrode. To dissolve the AAO template after plating, the samples were immersed in $0.5 \mathrm{M} \mathrm{KOH}\left(50 \%\right.$, BASF) for $30 \mathrm{~min}$ at $30^{\circ} \mathrm{C}$, followed by rinsing with $\mathrm{H}_{2} \mathrm{O}$. To fabricate the flexible nanomesh sheets, prior to electrodeposition of nickel, a thin layer of copper was electroplated from $1 \mathrm{M}$ copper(II) sulfate (99\%, Merck) $+1 \mathrm{M}$ sulfuric acid $(96 \%, \mathrm{KMG})$ bath at $-10 \mathrm{~mA} / \mathrm{cm}^{2}$ and $30{ }^{\circ} \mathrm{C}$ for $20 \mathrm{~s}$. The subsequent nickel plating was carried out from nickel sulfamate / boric acid bath, under the same galvanostatic conditions. Upon plating for $300 \mathrm{~s}$, the color of the sample changed from black to metallic grey, indicating complete filling of the AAO pores. From this point, electrodeposition was continued at $50 \mathrm{~mA} / \mathrm{cm}^{2}$ for $200-500 \mathrm{~s}$ to deposit a planar nickel layer above the nanowires. After that, the sample was immersed in $1 \mathrm{M} \mathrm{KOH}$ for $5 \mathrm{~min}$. During this time, the nanomesh sheet was observed to partially 
detach from the wafer support. The residual copper at the bottom end of the nanowire network was etched using saturated solution of potassium persulfate (99+\%, Sigma Aldrich). Then the sample was immersed in $0.5 \mathrm{M} \mathrm{KOH}$ for 30 min to remove any residual AAO template. After that, the sample was rinsed with copious amount of water and stored for later application. The complete scheme for the fabrication of the nanomesh is presented in the Figure S1.

Engineering of nanomeshes properties. To adjust the surface area and porosity of the nanomesh, the pore distribution of AAO templates was modified by either changing the potential during anodization or by introducing an additional pore widening etch prior to electrodeposition of nickel. To avoid excessive pore widening during anodization at higher potentials, the temperature during anodization was adjusted depending on the anodization voltage: $30{ }^{\circ} \mathrm{C}(\mathrm{U} \leq 55 \mathrm{~V}), 15{ }^{\circ} \mathrm{C}$ $(\mathrm{U}=70 \mathrm{~V}), 0{ }^{\circ} \mathrm{C}(\mathrm{U}=85 \mathrm{~V})$ and $-5^{\circ} \mathrm{C}(100 \mathrm{~V})$. Pore widening was performed by immersing the templates in 5\% wt. solution of o-phosphoric acid (85\%, Honeywell) for the time periods of 5-20 min at $30{ }^{\circ} \mathrm{C}$, prior to $\mathrm{TiO}_{2}$ etching.

Functionalization with platinum for HER. To enhance the activity of the nickel nanomesh and the nickel foam for HER, after the initial HER tests of both materials they were coated with platinum nanoparticles. First, the samples were immersed in a $1 \mathrm{mM}$ solution of hexachloroplatinic acid (99.995\%, Sigma-Aldrich) for $30 \mathrm{~s}$, followed by washing with copious amount of water. Then, the samples were brought into the $1 \mathrm{M} \mathrm{KOH}$ electrolyte and polarized at $-0.2 \mathrm{~V} v \mathrm{~s}$. RHE for 1 min to reduce the chemisorbed platinum species. The entire procedure was repeated 3 times. 
Composition analysis. Crystalline purity of the flexible nanomesh was analyzed with Grazing Incidence XRD, using PANalytical X'Pert MRD diffractometer equipped with $\mathrm{Cu} \mathrm{K} \alpha$ radiation $(\lambda=1.540598 \AA)$ at the incidence angle $\Omega$ of $1^{\circ}$ in the $2 \theta$ range of $10-80^{\circ}$ with step size of $0.020^{\circ}$ and sampling time of $12.25 \mathrm{~s} / \mathrm{step}$.

Loading of the platinum on the nanomesh was determined by EDX measurement of the cross section of the nanomesh $(6 \mu \mathrm{m} \times 4 \mu \mathrm{m})$, using electron beam operating at $20 \mathrm{keV}$. The loading of platinum was derived from the atomic ratio between $\mathrm{Pt}$ and Ni (estimated as 0.026), using the known quantity of Ni present in the $4.75 \mu \mathrm{m}$ thick, $76 \%$ porous nanomesh of $17.3 \mu \mathrm{mol}(\mathrm{Ni}) / \mathrm{cm}^{2}$. The measurements were performed on 19 different spots of the sample and the results were averaged.

Determination of nanomesh porosity. The total porosity of the Si-supported nanomesh was determined by comparing the electrodeposition rate of the networks, to that expected for a dense nickel film electroplated under the same current density. The electrodeposition rate of the nanomesh was determined with SEM, by measuring the network thickness across the cross section of five samples plated for different amount of times. The relation used to determine the porosity is described by the Equation 3:

$$
P=100 \%\left(1-r_{\text {bulk }} / r_{\text {nm }}\right)
$$

where $P$ is the porosity of the nanomesh, $r_{\text {bulk }}$ is the growth rate of bulk nickel film, calculated with the Faraday's law of electrolysis (accounting for the 95\% nickel plating efficiency) and $r_{\mathrm{nm}}$ is the growth rate of the nanomesh (for exemplary analysis of porosity, see Figure S6). 
Determination of nanomesh surface area. The characterization of the surface area was done with cyclic voltammetry (CV) and electrochemical impedance spectroscopy (EIS) in a nitrogensaturated $2 \mathrm{M}$ aqueous $\mathrm{KOH}$ solution, in accordance to the previously published methodology. ${ }^{57,58}$ The measurements were done using Autolab PGSTAT301 potentiostat/galvanostat and a $\mathrm{Hg} / \mathrm{HgO}$ reference electrode (IJ Cambria Scientific), measuring the potential near the sample surface through a Luggin capillary. The potentials are referenced to the reversible hydrogen electrode $R H E$ $(-0.938 \mathrm{~V}$ vs. $\mathrm{Hg} / \mathrm{HgO}$ in $2 \mathrm{M} \mathrm{KOH})$. Each experiment was performed in a freshly replaced electrolyte and repeated at least 3 times. Cyclic voltammetry measurements were done in the potential range of $-0.30 \mathrm{~V}$ and $+0.55 \mathrm{~V}$ and a scan speed of $50 \mathrm{mV} / \mathrm{s}$, recording 3 scans each time. The electrochemical impedance spectroscopy was performed at $-0.17 \mathrm{~V} v s$. RHE by applying an AC signal with a $10 \mathrm{mV}$ amplitude in the $5 \mathrm{kHz}-0.5 \mathrm{~Hz}$ frequency range. As a reference, experiments were performed under the same conditions on a planar nickel foil, freshly polished to mirror quality. The relative surface area of the polished planar foil (which is the ratio between the real and geometrical surface area) had been verified as 1.005 by Atomic Force Microscopy (Brüker Dimension Icon). The footprint-normalized surface area of the nanomesh was calculated as the ratio of the $\mathrm{Ni}(\mathrm{OH})_{2}$ formation charge $(\mathrm{CV})$ or the double layer capacitance (EIS), measured on the nanomesh to those measured on the planar foil (for exemplary CV and EIS spectra, see Fig S6). The double layer capacitance was derived from the EIS spectra according to the method of Grden et $a l .{ }^{58}$. For the nanomesh fabricated from $40 \mathrm{~V}$ - anodized AAO, five samples of increasing network thickness were analyzed. The volumetric surface area of such nanomesh was calculated as the linear slope of the footprint-normalized surface area (averaged from the CV and EIS for each sample) versus the network thickness, measured with SEM. The surface area of the nanomeshes fabricated from the modified AAO templates was analyzed only with cyclic 
voltammetry (the results from CV and EIS in the previous experiments were found to be similar), under the same experimental conditions. For such meshes, the volumetric surface area was calculated as the ratio between the footprint-normalized surface area and the nanomesh thickness, measured across each sample with SEM.

To cross-validate the electrochemically-determined surface area, $\mathrm{Kr}$ adsorption was performed on the nanomesh fabricated from $40 \mathrm{~V}$ AAO. Five wafer-supported nanomesh samples (each having $3.3 \mu \mathrm{m}$-thick network) were measured simultaneously to be above the detection limit $\left(0.1 \mathrm{~m}^{2}\right)$. The measurement was done at $77 \mathrm{~K}$ and the BET method was applied in the $0.003-0.005 \mathrm{p} / \mathrm{p}^{0}$ range, according to the previously described methodology. ${ }^{59}$ Prior to the measurement, the samples were degassed in vacuo at $110^{\circ} \mathrm{C}$ for $12 \mathrm{~h}$. After the measurement, the results were corrected for the sample area not covered with nanomeshes (approx. $11 \mathrm{~cm}^{2}$ each) and normalized per sample.

Hydrogen evolution study. The measurements of hydrogen evolution reaction were done in $1 \mathrm{M}$ $\mathrm{KOH}$ solution, thermostated at $21{ }^{\circ} \mathrm{C}$. The tests were performed in a $600 \mathrm{~mL}$ jacketed glass cell, having the compartments for the working and the counter electrode separated by a glass tube with a glass frit membrane to avoid the diffusion of the gases or trace Pt between the electrodes (the consecutive LSV scans did not show any increase of the current density at any of the analyzed working electrodes). The HER tests were performed on a flexible nickel nanomesh fabricated from $40 \mathrm{~V}$ - anodized AAO, as well as on a commercial high surface area nickel foam (Ni-5763, Recemat B.V.) and a PTFE-coated platinized Vulcan carbon cloth with a microporous carbon layer $\left(0.2 \mathrm{mg} \mathrm{Pt} / \mathrm{cm}^{2}=20 \%\right.$ wt. Pt, FuelCellsEtc). For the measurements, the samples were cut into coupons of $2.0 \times 1.0 \mathrm{~cm}$ and connected with a crocodile clamp to the potentiostat (Autolab PGSTAT301). Prior to the measurements, the electrolyte in the working electrode compartment 
was saturated with hydrogen by electrolysis at the current of $1 \mathrm{~A}$ for $10 \mathrm{~min}$, using titanium mesh working electrode. All the following measurements were performed using 3 electrode setup, where the sample was connected as the working electrode in the working electrode compartment and a platinum mesh as a counter electrode in the counter electrode compartment. The reference $\mathrm{Hg} / \mathrm{HgO}$ electrode was positioned $5 \mathrm{~mm}$ in front of the samples surface. Prior to the measurements, the potential of the reference electrode was validated as $-0.920 \mathrm{~V}$ vs. RHE, using commercial reversible hydrogen electrode (Gaskatel). During the measurements, the samples were contacted from the top, maintaining minimal immersion $(<1 \mathrm{~mm})$ of the metal contacts in the electrolyte. Linear scan voltammetry was performed at $1 \mathrm{mV} / \mathrm{s}$, with the correction for $90 \%$ of the uncompensated resistance (iR). Prior to each LSV scan, the iR drop of the system was determined with electrochemical impedance spectroscopy, at the potential of $-0.2 \mathrm{~V} v s$. RHE, in the frequency range of $10 \mathrm{kHz}-0.5 \mathrm{~Hz}$, using the $\mathrm{AC}$ excitation potential of $10 \mathrm{mV}$. The $\mathrm{iR}$ drop was derived from the high frequency intercept of the semicircles fitted to the complex impedance spectra. ${ }^{44}$ For the nickel materials, the iR drop was in the range of $200-300 \mathrm{~m} \Omega$, and for the platinized carbon about $1 \Omega$. For each sample, three LSV scans were recorded and averaged. Constant polarization experiments were performed at $-0.2 \mathrm{~V} v s$. RHE for $10 \mathrm{~min}$, without iR drop compensation. Longterm stability was assessed at $-0.2 \mathrm{~V}$ vs. RHE for $30 \mathrm{~h}$ of HER without $\mathrm{iR}$ drop compensation. Mild agitation of the electrolyte was applied by a magnetic stirrer to lower the buildup of temperature and concentration in the vicinity of the working electrode. After $18 \mathrm{~h}$ of measurement, the samples were allowed to rest for $1 \mathrm{~h}$ at their open circuit potential, followed by reapplication of the constant potential of $-0.2 \mathrm{~V}$ vs. RHE for the following $12 \mathrm{~h}$. 
Statistical analysis. All the statistical analysis was performed using Origin 2017. The analysis of the structural parameters of the nanomesh (nanowire diameter, interwire spacing, nanomesh pore size) prepared from $40 \mathrm{~V}$ - anodized $\mathrm{AAO}$ was performed with a sample size $\mathrm{N}=550$ for each of the parameters. The analysis of the structure of the nanomeshes prepared from other AAO templates was performed with a typical sample size $\mathrm{N}>30$ for each of the parameters. The $95 \%$ confidence intervals $(95 \% \mathrm{CI})$ of the model $V S A$ and porosity reported in the text were calculated with the partial derivative method, using $95 \% \mathrm{CI}$ of the nanowire diameter and spacing determined with SEM. The $95 \% \mathrm{CI}$ of experimentally-determined porosity and $V S A$ for the nanomesh produced from $40 \mathrm{~V}$ - AAO were calculated with error-weighted linear regression analysis - the weights were the $95 \% \mathrm{CI}$ of the nanomesh thickness, $\mathrm{Ni}(\mathrm{OH})_{2}$ formation charges and of the nickel double layer capacitance. For the nanomeshes produced from other AAO templates, the $95 \% \mathrm{CI}$ of experimental porosity and $V S A$ were determined with partial derivative method. The sampling size of nanomesh thickness was $\mathrm{N}>100$ for each of the nanomesh sample. For each of the nanomesh sample, the total sampling size of $\mathrm{Ni}(\mathrm{OH})_{2}$ formation charges and nickel double layer capacitance was $\mathrm{N}=18$.

\section{ASSOCIATED CONTENT}

\section{Supporting Information.}

Derivation of the Equations 1 and 2. Tables showing structural properties of the nanomeshes prepared in this work and the reference data on porosity and surface area of various porous metals from the literature. Figures showing general fabrication scheme of the nanomesh, nanowire and pore size histograms, different magnification images of the networks, XRD pattern of flexible nanomesh, detailed geometrical representation of the nanomesh unit cell, nanomesh thickness vs. 
plating time, nanomesh surface area and its theoretical dependence on the nanowire diameter, nanomesh structures prepared from different AAO templates, EDX spectrum of Pt-coated nanomesh and photograph of the tested materials during HER, cyclic voltammogram of the nanomesh after long-term HER. This material is available free of charge via the Internet at http://pubs.acs.org.

\section{AUTHOR INFORMATION}

\section{Corresponding Author}

*E-mail: stanislaw.zankowski@imec.be

\section{ORCID}

Stanislaw P. Zankowski: 0000-0001-8616-1849

Philippe M. Vereecken: 0000-0003-4115-0075

\section{Author Contributions}

Both authors contributed equally to the design of the synthesis and characterization of the material. The fabrication and characterization of the material, together with the derivation of the mathematical model and testing for hydrogen evolution was performed by S.P.Z. The manuscript was written by S.P.Z. and revised by P.M.V. All authors have given approval to the final version of the manuscript.

\section{ACKNOWLEDGMENTS}


S.P.Z wishes to acknowledge the PhD funding from imec. The authors wish to express their acknowledgements to T. Stassin and I. Stassen (Kr adsorption measurements), H. Philipsen (hardware for the $\mathrm{H}_{2}$ evolution study), O. Richard (TEM) and S. Calderon Ardila (AFM). We also want to thank Recemat B.V. for providing samples of nickel foam. Furthermore, we want to acknowledge the ESTORE team at imec for reviewing the mathematical model and the fruitful discussions during preparation of this work.

\section{REFERENCES}

(1) Dörfelt, C.; Kolvenbach, R.; Wirth, A. S.; Albert, M.; Köhler, K. Catalytic Properties of a Novel Raney-Nickel Foam in the Hydrogenation of Benzene. Catal. Letters 2016, 146, $2425-2429$.

(2) Khosravanipour Mostafazadeh, A.; Zolfaghari, M.; Drogui, P. Electrofiltration Technique for Water and Wastewater Treatment and Bio-Products Management: A Review. J. Water Process Eng. 2016, 14, 28-40.

(3) Yuan, W.; Tang, Y.; Yang, X.; Wan, Z. Porous Metal Materials for Polymer Electrolyte Membrane Fuel Cells - A Review. Appl. Energy 2012, 94, 309-329.

(4) Yang, G. F.; Song, K. Y.; Joo, S. K. A Metal Foam as a Current Collector for High Power and High Capacity Lithium Iron Phosphate Batteries. J. Mater. Chem. A 2014, 2, 1964819652.

(5) Xu, M.; Xu, R.; Zhao, Y.; Chen, L.; Huang, B.; Wei, W. Hierarchically Porous Ni Monolith@branch-Structured NiCo2O4 for High Energy Density Supercapacitors. Prog. 
Nat. Sci. Mater. Int. 2016, 26, 276-282.

(6) Zhu, W.; Zhang, R.; Qu, F.; Asiri, A. M.; Sun, X. Design and Application of Foams for Electrocatalysis. ChemCatChem 2017, 9, 1721-1743.

(7) Li, X.; Lu, X.; Kan, X. 3D Electrochemical Sensor Based on Poly(Hydroquinone)/Gold Nanoparticles/Nickel Foam for Dopamine Sensitive Detection. J. Electroanal. Chem. 2017, $799,451-458$.

(8) Pikul, J. H.; Gang Zhang, H.; Cho, J.; Braun, P. V.; King, W. P. High-Power Lithium Ion Microbatteries from Interdigitated Three-Dimensional Bicontinuous Nanoporous Electrodes. Nat. Commun. 2013, 4, 1732.

(9) Kumar, R.; Bhuvana, T.; Rai, P.; Sharma, A. Highly Sensitive Non-Enzymatic Glucose Detection Using 3-D Ni3(VO4)2 Nanosheet Arrays Directly Grown on Ni Foam. J. Electrochem. Soc. 2018, 165, B1-B8.

(10) Schomburg, W. K. Scaling Laws. In Introduction to Microsystem Design; Springer-Verlag, $2011 ;$ p 3.

(11) Kjeang, E.; Djilai, N.; Sinton, D. Advances in Microfluidic Fuel Cells. In Micro Fuel Cells: Principles and Applications; Zhao, T. S., Ed.; Elsevier Inc., 2009; pp 99-105.

(12) Hamelers, B.; Sleutels, T. H. J. .; Jeremiasse, A. W.; Post, J. W.; Strik, D. P. B. T. B.; Rozendal, R. A. Technological Factors Affecting BES Performance and Bottlenecks Towards Scale Up. In Bioelectrochemical Systems: From Extracellular Electron Transfer to Biotechnological Application; Rabaey, K., Agenent, L., Schroder, U., Keller, J., Eds.; 
IWA Publishing, 2010; p 220.

(13) Gerasopoulos, K.; Pomerantseva, E.; McCarthy, M.; Brown, A.; Wang, C.; Culver, J.; Ghodssi, R. Hierarchical Three-Dimensional Microbattery Electrodes Combining Bottomup Self-Assembly and Top-down Micromachining. ACS Nano 2012, 6, 6422-6432.

(14) Wittstock, A.; Biener, J.; Bäumer, M. Chapter 1. Introduction to Nanoporous Gold. In Nanoporous Gold: From an Ancient Technology to a High-Tech Material; 2012; pp 1-10.

(15) Tappan, B. C.; Stephen A, S.; Luther, E. P. Nanoporous Metal Foams. Angew. Chemie - Int. Ed. 2010, 49, 4544-4565.

(16) Liu, P. S.; Chen, G. F. Application of Porous Metals. In Porous materials. Processing and Applications; Elsevier Inc., 2014; pp 113-188.

(17) Li, L.; Fan, W.; Xuan, J.; Leung, M. K. H. Dimensionless Parametric Sensitivity Analysis of Microfluidic Fuel Cell with Flow-through Porous Electrodes. Electrochim. Acta 2016, $187,636-645$.

(18) Yue, Y.; Liang, H. 3D Current Collectors for Lithium-Ion Batteries: A Topical Review. Small Methods 2018, 2, 1800056.

(19) Ye, J.; Baumgaertel, A. C.; Wang, Y. M.; Biener, J.; Biener, M. M. Structural Optimization of 3D Porous Electrodes for High-Rate Performance Lithium Ion Batteries. ACS Nano 2015, 9, 2194-2202.

(20) Juarez, T.; Biener, J.; Weissmüller, J.; Hodge, A. M. Nanoporous Metals with Structural Hierarchy: A Review. Adv. Eng. Mater. 2017, 19, 1-23. 
(21) Molchan, I. S.; Molchan, T. V.; Gaponenko, N. V.; Skeldon, P.; Thompson, G. E. ImpurityDriven Defect Generation in Porous Anodic Alumina. Electrochem. commun. 2010, 12, 693-696.

(22) Vanpaemel, J.; Abd-Elnaiem, A. M.; De Gendt, S.; Vereecken, P. M. The Formation Mechanism of 3D Porous Anodized Aluminum Oxide Templates from an Aluminum Film with Copper Impurities. J. Phys. Chem. C 2015, 119, 2105-2112.

(23) Wang, W.; Tian, M.; Abdulagatov, A.; George, S. M.; Lee, Y. C.; Yang, R. ThreeDimensional Ni/TiO2 Nanowire Network for High Areal Capacity Lithium Ion Microbattery Applications. Nano Lett. 2012, 12, 655-660.

(24) Wen, R.; Xu, S.; Ma, X.; Lee, Y. C.; Yang, R. Three-Dimensional Superhydrophobic Nanowire Networks for Enhancing Condensation Heat Transfer. Joule 2018, 2, 269-279.

(25) Tian, M.; Wang, W.; Wei, Y.; Yang, R. Stable High Areal Capacity Lithium-Ion Battery Anodes Based on Three-Dimensional Ni-Sn Nanowire Networks. J. Power Sources 2012, $211,46-51$.

(26) Tian, M.; Wang, W.; Liu, Y.; Jungjohann, K. L.; Thomas Harris, C.; Lee, Y. C.; Yang, R. A Three-Dimensional Carbon Nano-Network for High Performance Lithium Ion Batteries. Nano Energy 2015, 11, 500-509.

(27) Martín, J.; Martín-González, M.; Francisco Fernández, J.; Caballero-Calero, O. Ordered Three-Dimensional Interconnected Nanoarchitectures in Anodic Porous Alumina. Nat. Commun. 2014, 5, 1-9. 
(28) Lee, W.; Park, S.-J. Porous Anodic Aluminum Oxide: Anodization and Templated Synthesis of Functional Nanostructures. Chem. Rev. 2014, 114, 7487-7556.

(29) Li, A. P.; Müller, F.; Birner, A.; Nielsch, K.; Gösele, U. Hexagonal Pore Arrays with a 50420 Nm Interpore Distance Formed by Self-Organization in Anodic Alumina. J. Appl. Phys. $1998,84,6023-6026$.

(30) Tang, M. H.; Hahn, C.; Klobuchar, A. J.; Ng, J. W. D.; Wellendorff, J.; Bligaard, T.; Jaramillo, T. F. Nickel-Silver Alloy Electrocatalysts for Hydrogen Evolution and Oxidation in an Alkaline Electrolyte. Phys. Chem. Chem. Phys. 2014, 16, 19250-19257.

(31) Chen, A. Y.; Qiu, Y. J.; Zhu, Y. K.; Wang, D.; Yang, J. H.; Wang, X. Y.; Xie, X. F. Facile Fabrication of Nanoporous Gold with Bimodal Pore Structure. Mater. Lett. 2016, 184, 282 285.

(32) Rösner, H.; Parida, S.; Kramer, D.; Volkert, C. A.; Weissmüller, J. Reconstructing a Nanoporous Metal in Three Dimensions: An Electron Tomography Study of Dealloyed Gold Leaf. Adv. Eng. Mater. 2007, 9, 535-541.

(33) Leventis, N.; Chandrasekaran, N.; Sotiriou-Leventis, C.; Mumtaz, A. Smelting in the Age of Nano: Iron Aerogels. J. Mater. Chem. 2009, 19, 63-65.

(34) Krishna, K. S.; Sandeep, C. S. S.; Philip, R.; Eswaramoorthy, M. Mixing Does the Magic: A Rapid Synthesis of High Surface Area Noble Metal Nanosponges Showing Broadband Nonlinear Optical Response. ACS Nano 2010, 4, 2681-2688.

(35) Vineesh, T. V.; Mubarak, S.; Hahm, M. G.; Prabu, V.; Alwarappan, S.; Narayanan, T. N. 
Controllably Alloyed, Low Density, Free-Standing Ni-Co and Ni-Graphene Sponges for Electrocatalytic Water Splitting. Sci. Rep. 2016, 6, 31202.

(36) Hales, T. C. The Honeycomb Conjecture. Discrete Comput. Geom. 2001, 25, 1-22.

(37) Biener, J.; Hodge, A. M.; Hamza, A. V. Microscopic Failure Behavior of Nanoporous Gold. Appl. Phys. Lett. 2005, 87, 1-3.

(38) Lambers, E. S.; Dykstal, C. N.; Seo, J. M.; Rowe, J. E.; Holloway, P. H. Room-Temperature Oxidation of Ni(110) at Low and Atmospheric Oxygen Pressures. Oxid. Met. 1996, 45, 301321.

(39) Medway, S. L.; Lucas, C. A.; Kowal, A.; Nichols, R. J.; Johnson, D. In Situ Studies of the Oxidation of Nickel Electrodes in Alkaline Solution. J. Electroanal. Chem. 2006, 587, $172-$ 181.

(40) Lee, W.; Ji, R.; Gösele, U.; Nielsch, K. Fast Fabrication of Long-Range Ordered Porous Alumina Membranes by Hard Anodization. Nat. Mater. 2006, 5, 741-747.

(41) Tripathy, J.; Wiley, J. B. Fabrication of Thick Porous Anodized Aluminum Oxide Templates. J. Solid State Electrochem. 2015, 19, 1447-1452.

(42) Blanco, H.; Faaij, A. A Review at the Role of Storage in Energy Systems with a Focus on Power to Gas and Long-Term Storage. Renew. Sustain. Energy Rev. 2018, 81, 1049-1086.

(43) Pierozynski, B.; Mikolajczyk, T. Cathodic Evolution of Hydrogen on Platinum-Modified Nickel Foam Catalyst. Electrocatalysis 2015, 7, 121-126.

(44) Oelßner, W.; Berthold, F.; Guth, U. The IR Drop - Well-Known but Often Underestimated 
in Electrochemical Polarization Measurements and Corrosion Testing. Mater. Corros. 2006, $57,455-466$.

(45) Yang, C.; Zhang, Q. B.; Abbott, A. P. Facile Fabrication of Nickel Nanostructures on a Copper-Based Template via a Galvanic Replacement Reaction in a Deep Eutectic Solvent. Electrochem. commun. 2016, 70, 60-64.

(46) You, B.; Jiang, N.; Sheng, M.; Gul, S.; Yano, J.; Sun, Y. High-Performance Overall Water Splitting Electrocatalysts Derived from Cobalt-Based Metal-Organic Frameworks. Chem. Mater. 2015, 27, 7636-7642.

(47) Wu, H. Bin; Xia, B. Y.; Yu, L.; Yu, X. Y.; Lou, X. W. Porous Molybdenum Carbide NanoOctahedrons Synthesized via Confined Carburization in Metal-Organic Frameworks for Efficient Hydrogen Production. Nat. Commun. 2015, 6, 1-8.

(48) Chen, W.; Pei, J.; He, C. T.; Wan, J.; Ren, H.; Zhu, Y.; Wang, Y.; Dong, J.; Tian, S.; Cheong, W. C.; et al. Rational Design of Single Molybdenum Atoms Anchored on N-Doped Carbon for Effective Hydrogen Evolution Reaction. Angew. Chemie - Int. Ed. 2017, 56, $16086-16090$.

(49) Feng, J. X.; Wu, J. Q.; Tong, Y. X.; Li, G. R. Efficient Hydrogen Evolution on Cu NanodotsDecorated Ni3S2 Nanotubes by Optimizing Atomic Hydrogen Adsorption and Desorption. J. Am. Chem. Soc. 2018, 140, 610-617.

(50) Feng, J. X.; Xu, H.; Dong, Y. T.; Lu, X. F.; Tong, Y. X.; Li, G. R. Efficient Hydrogen Evolution Electrocatalysis Using Cobalt Nanotubes Decorated with Titanium Dioxide Nanodots. Angew. Chemie - Int. Ed. 2017, 56, 2960-2964. 
(51) Chen, W.; Pei, J.; He, C. T.; Wan, J.; Ren, H.; Wang, Y.; Dong, J.; Wu, K.; Cheong, W. C.; Mao, J.; et al. Single Tungsten Atoms Supported on MOF-Derived N-Doped Carbon for Robust Electrochemical Hydrogen Evolution. Adv. Mater. 2018, 30, 1800396.

(52) Abouatallah, R. M.; Kirk, D. W.; Thorpe, S. J.; Graydon, J. W. Reactivation of Nickel Cathodes by Dissolved Vanadium Species during Hydrogen Evolution in Alkaline Media. Electrochim. Acta 2001, 47, 613-621.

(53) Hall, D. S.; Bock, C.; Macdougall, B. R. The Electrochemistry of Metallic Nickel : Oxides , Hydroxides , Hydrides and Alkaline Hydrogen Evolution. J. Electrochem. Soc. 2013, 160, $235-243$.

(54) Janssen, L. J. J.; Barendrecht, E. Electrolytic Resistance of Solution Layers at Hydrogen and Oxygen Evolving Electrodes in Alkaline Solution. Electrochim. Acta 1983, 28, 341346.

(55) Janssen, L. J. J.; Sillen, C. W. M. P.; Barendrecht, E.; van Stralen, S. J. D. Bubble Behaviour during Oxygen and Hydrogen Evolution at Transparent Electrodes in $\mathrm{KOH}$ Solution. Electrochim. Acta 1984, 29, 633-642.

(56) Park, S.-H.; Kim, S.; Lee, D.-J.; Yun, S.; Khim, Z. G.; Kim, K.-B. Selective Wet-Chemical Etching of the Barrier Layer during Formation of Porous Anodic Aluminum Oxide Template. J. Electrochem. Soc. 2009, 156, K181.

(57) Van Drunen, J.; Kinkead, B.; Wang, M. C. P.; Sourty, E.; Gates, B. D.; Jerkiewicz, G. Comprehensive Structural, Surface-Chemical and Electrochemical Characterization of Nickel-Based Metallic Foams. ACS Appl. Mater. Interfaces 2013, 5, 6712-6722. 
(58) Grdeń, M.; Alsabet, M.; Jerkiewicz, G. Surface Science and Electrochemical Analysis of Nickel Foams. ACS Appl. Mater. Interfaces 2012, 4, 3012-3021.

(59) Stassen, I.; Styles, M.; Grenci, G.; Gorp, H. Van; Vanderlinden, W.; De Feyter, S.; Falcaro, P.; De Vos, D.; Vereecken, P.; Ameloot, R. Chemical Vapour Deposition of Zeolitic Imidazolate Framework Thin Films. Nat. Mater. 2016, 15, 304-312. 


\section{ToC Graphics:}
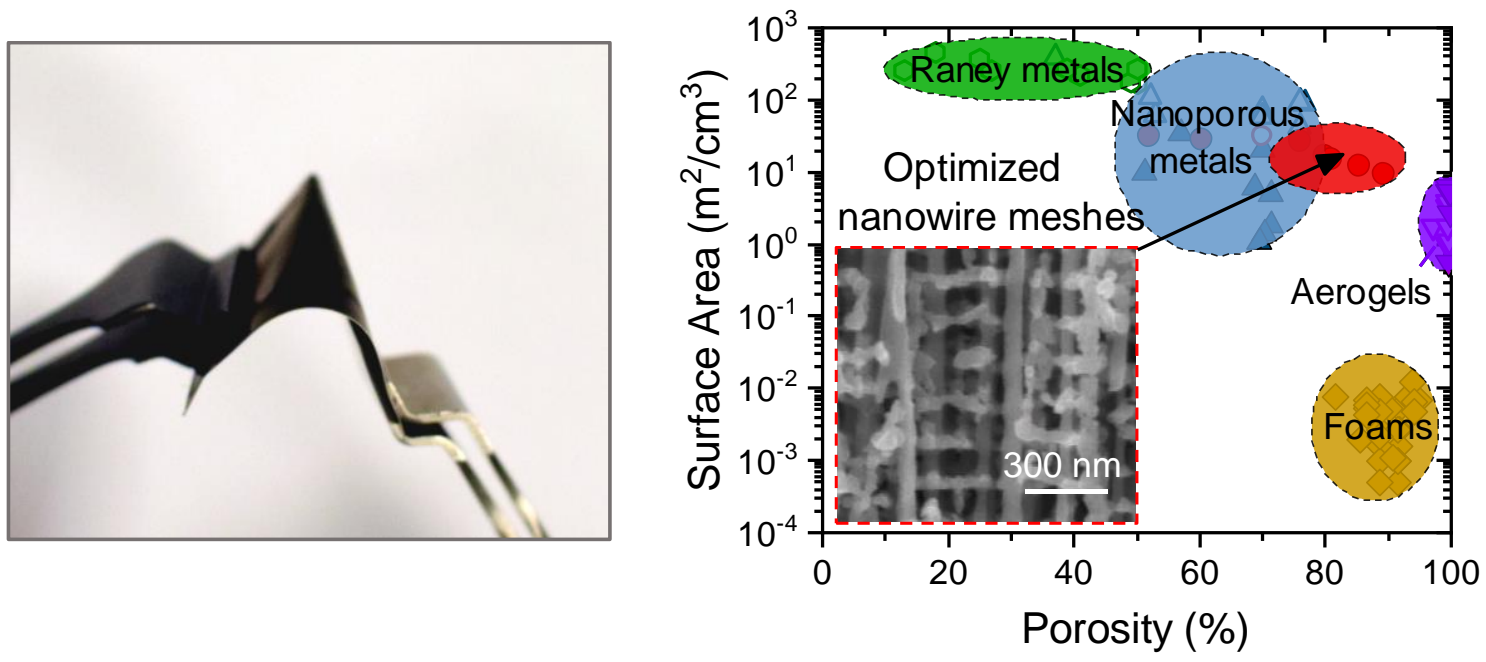